\title{
OUTCOME OF DOUBLE NERVE TRANSFER CLOSE TO TARGET MUSCLE BOTH FOR SHOULDER ABDUCTION AND ELBOW FLEXION IN EARLY C5 -C6 BRACHIAL PLEXUS INJURY.
}

1. FCPS

AP Plastic Surgery

Rawalian Burn and Reconstructive

Surgery

Holy Family Hospital Rawalpindi.

Ex Hand Fellow National Orthopedic

Hospital Bahawalpur.

2. FCPS

Associate Professor Orthopedics

Gomal Medical College,

Dera Ismail Khan.

Ex Hand Fellow National Orthopedic

Hospital Bahawalpur.

3. FCPS

AP Plastic Surgery

Aziz Bhatti Shaheed Teaching

Hospital Gujrat.

Ex Hand Fellow National Orthopedic Hospital Bahawalpur.

4. Diplomat American Board

Orthopedic Surgery

Professor of Orthopedics

National Orthopedic Hospital

Bahawalpur.

Correspondence Address:

Dr. Husnain Khan

Rawalian Burn and Reconstructive Surgery

Holy Family Hospital Rawalpindi.

Ex Hand Fellow National Orthopedic

Hospital

Bahawalpur

hasnainkhan113@gmail.com

Article received on:

21/10/2019

Accepted for publication:

$13 / 02 / 2020$

\section{Husnain Khan ${ }^{1}$, Muhammad Shafique ${ }^{2}$, Zahid Iqbal Bhatti ${ }^{3}$, Tehseen Ahmad Cheema}

ABSTRACT... Adult brachial plexus injury is a now a common problem due to high incidence of motorbike accidents. Among all types, C 5 and C6 (upper brachial plexus injury) is the most common. If the patient present within 6 months then nerve transfer is the preferred treatment. However, there are different options for nerve transfer and different approaches for surgery. Objectives: The objective of the study was to share our experience of nerve transfer close to target muscles in upper brachial plexus injury. Study Design: Quaisi experimental study. Setting: National Orthopaedic Hospital, Bahawalpur. Period: January 2015 to June 2018. Material \& Methods: Total 32 patients were operated with isolated C5 and C6 injury. In all patients four nerve transfers were done. For shoulder abduction posterior approach was used and accessory to suprascapular nerve and one of motor branch of radial to axillary nerve were transferred. Modified Oberlin transfer was done for elbow flexion. Both shoulder abduction and elbow flexion was graded according to medical research council grading system. Results: After one year follow up more than $75 \%$ of the patients showed good to normal shoulder abduction and $\mathbf{8 7 . 5 0 \%}$ showed good to normal elbow flexion. Residual Median nerve damage was noted only in two patients $(6.25 \%)$. Conclusion: If there is no evidence of recovery up to three months early nerve transfer should be considered, ideal time is 3-6 months. Nerve transfer close to target muscle yields superior results. The shoulder stabilizers and abductors should ideally be innervated by double nerve transfer through posterior approach. Similarly double fascicular transfer (modified Oberlin) should be done for elbow flexion.

\section{Key words: $\quad$ Elbows Flexion, Shoulder Stabilization, Upper Brachial Plexus Injury.}

Article Citation: Khan H, Shafique M, Bhatti Zl, Cheema TA. Outcome of double nerve transfer close to target muscle both for shoulder abduction and elbow flexion in early C5 -C6 Brachial Plexus injury. Professional Med J 2020; 27(7):1442-1447. DOI: 10.29309/TPMJ/2020.27.07.4269

\section{INTRODUCTION}

Due to increase in high-velocity motor vehicle accidents the frequency of brachial plexus injuries is progressively increasing. Among all motorcycle accidents, $5 \%$ results in brachial plexus injuries, however, $84 \%$ of these injuries are caused by the motorcycle collisions. ${ }^{1}$ If one looks into the pattern of injury, $75 \%$ of those are supraclavicular and $25 \%$ are infraclavicular. All the roots from C4-T1 are involved in supraclavicular injuries in75-80\% of the cases, mostly being $\mathrm{C} 5$ C6 rupture followed by $\mathrm{C} 7-\mathrm{C} 8-\mathrm{T} 1$ root avulsion from the spinal cord. $\mathrm{C} 5-\mathrm{C} 6$ or $\mathrm{C} 5-\mathrm{C} 6-\mathrm{C} 7$ roots are involved in $20-25 \%$ of supraclavicular injuries and C8-T1 in 2-3\% of injuries. ${ }^{2}$ In isolated C5- C6 lesions, aims of reconstructions are elbow flexion and shoulder stabilization, provided there is no evidence of recovery after 2-3 months.

Nerve transfer are the main stay of treatment if the patient present within 6 months of injury. Although the use of extra plexus motor donors such as ipsi-lateral cervical plexus, intercostal nerves ${ }^{3,4}$, the spinal accessory nerve in neck, the medial pectoral nerve $e^{5,6,7}$, the phrenic nerve ${ }^{8}$ had been advocated by different surgeons, but most recently there has been an increasing trend towards nerve transfer close to target muscles. As shoulder abduction and stabilization is maintained by supra-scapular and axillary nerves, researchers found better results with close to target muscle approach by using transfer of spinal accessory nerve to supra-scapular nerve and one of motor branch of radial nerve to the 
axillary nerve, instead of traditional nerve transfer in the neck. ${ }^{9}$ Similarly, technique of close to target muscle transfer in case of elbow flexion has been described as modified Oberlin technique. ${ }^{10}$ In this procedure one fascicle each from ulnar and median nerve is directly transferred to motor fascicles of bicep and brachialis muscles.

Extraplexus nerve transfer close to target muscle in early C5-6 brachial plexuses injury is a recent technique and not much work has been done to evaluate combination of double nerve transfer both for shoulder ( transfer of accessory nerve to suprascapular nerve and one of branch of radial to axillary nerve) and elbow (modified Oberlin transfer). We conducted this study to assess our results with close to target muscle nerve transfer using above mentioned nerve transfer in $\mathrm{C} 5$ and C 6 brachial plexus injury.

\section{MATERIAL \& METHODS}

It is a quasi-experimental study conducted at National Orthopaedic Hospital Bahawalpur from January 2015 to June 2018. After ethical approval, outcome of 32 patients was analysed with mean follow up of one year. All the patients with isolated C5-C6 injury were included in the study. Patients with $\mathrm{C} 5, \mathrm{C} 6$ and $\mathrm{C} 7$ injury, panbrachial plexus injury or nerve transfer in neck were excluded from the study. Patients presenting 10 months after trauma and obstetrical brachial plexus injuries were also excluded from the study. In all patients, surgery was done when there was no clinical and electrophysiological evidence of recovery. After informed consent same operative procedure was followed in all the patients.

\section{OPERATIVE PROCEDURE}

After general anaesthesia, the patient placed in lateral position and skin incision given on spine of scapula. (Figure-1) Dissection was carried out between the trapezius and supraspinatus muscle toward the suprascapular notch. Suprascapular nerve is identified after dividing suprascapular ligament. It is isolated as proximal as possible. After isolation of the suprascapular nerve, spinal accessory nerve is identified on under surface of the trapezius. It is divided as distally as possible after confirming its activity by nerve stimulator.
End-to-end neurorrhaphy is performed between the two nerves (Figure-2).

In same lateral position, incision is given between the deltoid and upper part of the triceps muscle. (Figure-3). Identification of teres major muscle is done, which is running from scapula to humerus in a transverse manner. The axillary nerve is first identified running on upper border of teres major, coming out of axilla to supply teres minor and the deltoid muscle. The nerve is dissected as proximal as possible. After that, radial nerve is exposed below the lower border of the teres major, in between the lower part of deltoid and upper part of triceps. One of the motor branches of the radial nerve to triceps, mostly branch to the medial head of the triceps is identified. Its activity is confirmed by nerve stimulator and dissecting out as distally as possible for a tension free end to end repair with proximal end of axillary nerve.

The patient is operated in supine position with arm fully abducted on the side table. The incision is made on the medial surface of the arm starting about $4 \mathrm{~cm}$ distal to the pectoralis major muscle lateral border. It is carried distally about $10 \mathrm{~cm}$ in between biceps and the triceps muscles. After identifying, musculoctaneous nerve, branches to biceps and the brachialis muscle are dissected as proximally as possible. The median and the ulnar nerve were identified. The fascia of the median nerve was opened near the branch to biceps and one of the motor fascicle of median nerve, preferably one supplying the flexor carpi radialis, was identified with the help of nerve stimulator and dissected as distally as possible to ensure a tension free repair with nerve to biceps muscle. Similarly one of the motor fascicles of the ulnar nerve was identified near the motor branch to brachialis muscle with the help of nerve stimulator. Same technique was applied for fascicular repair as shown in Figure-4.

\section{Post-Op care and follow-up}

The arm was immobilized in poly-sling for three weeks. The patient discharged after one day and stitches were removed after two weeks. Follow up was done at three months, 6 months and one year after surgery. Recovery of shoulder abductors 
and elbow flexors was graded according to Medical Research Counsel grading system. In this system, complete paralysis is graded as 0 (no power), flicker of contraction as 1 (trace), active movement with gravity eliminated as 2 (poor), active movement against gravity as 3 (fair), active movement against resistance as 4 (good) and normal movement as 5 (normal). Residual ulnar and median nerve deficit was also recorded.

Time of surgery since duration of injury was graded in 3 groups, i.e. early, intermediate and late. The patients operated within 3 months after injury were placed in early group, 3 to 6 months in intermediate and 6 to 10 months in late group. Age was also divided into 4 groups i.e. children, young, middle and old age. Patients of age 1-12 years were grouped under children, 13-40 years young, 41 to 60 years middle aged and more than 60 years old aged.

The data was analysed with SPSS ver. 20.

\section{RESULTS}

Out of 32 patients, 93.8\% $(n=30)$ were between the ages of $12-40$ years while $6.3 \%(n=2)$ were between the ages of 41-60 years. Thirty patients were male and two were females.

At one year follow up, 4 (12.5\%) patients showed normal shoulder abduction with complete range of movement and power $5 / 5,08(25 \%)$ of the patient showed fair shoulder abduction with shoulder abduction limited 90 degrees and power $3 / 5$ while most of the patient 20 (62.5\%) showed good shoulder abduction with abduction of 120 degrees and power $4 / 5$.

Results of elbow flexion were even better then shoulder abduction. Modified Oberlin transfer has resulted in good to normal elbow flexion in $\mathbf{8 7 . 5 0}$ $\%$ of the case. Out of these, $18.75 \%(n=6)$ patient should normal recovery with complete flexion and $5 / 5$ power at the elbow, $68.75 \%(n=22)$ showed $4 / 5$ muscle power, $9.375 \%(n=3)$ fair and $3.125 \%$ $(n=1)$ trace movement at the elbow.

Residual ulnar and median nerve damages were also recorded in these patient and only in two patients partial median nerve palsy was noted which recovered within six weeks possibly due to neuropraxia. Duration of injury i.e time since initial trauma up till the time of surgery was also recorded. $65.6 \%(n=21)$ of the patients were operated within3- 6 months after trauma. Six patients (18.8\%) were operated before 3 months, while five patients $(15.6 \%)$ were operated between six to ten months after trauma.

\begin{tabular}{|c|l|c|c|}
\hline \multicolumn{2}{|c|}{} & Frequency & Percent \\
\hline \multirow{3}{*}{ Valid } & $41-60$ YEARS & 2 & 6.3 \\
\cline { 2 - 4 } & $13-40$ YEARS & 30 & 93.8 \\
\cline { 2 - 4 } & Total & 32 & 100.0 \\
\hline \multicolumn{3}{|c|}{ Table-I. Age of the patient } \\
\hline \multicolumn{2}{|c|}{} \\
\hline
\end{tabular}

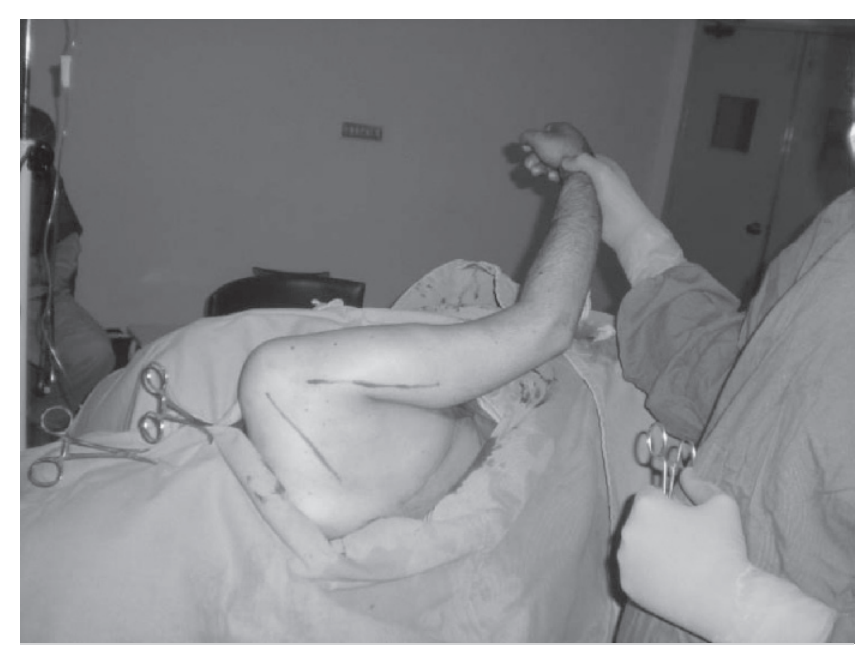

Figure-1. Marking of posterior approach for transfer of accessory to suprascapular and one of radial branch to axillary nerve

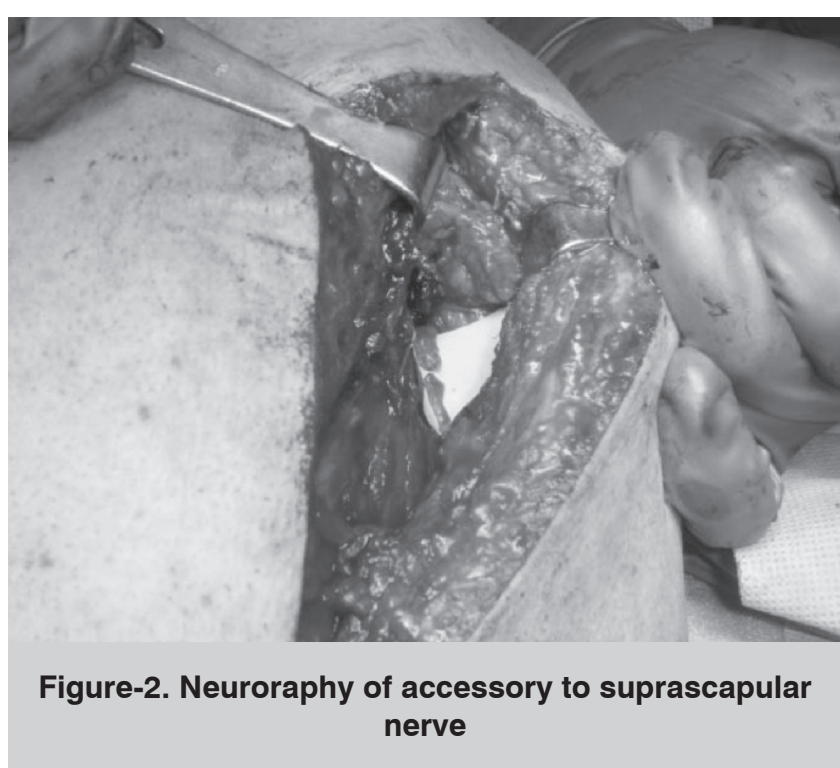



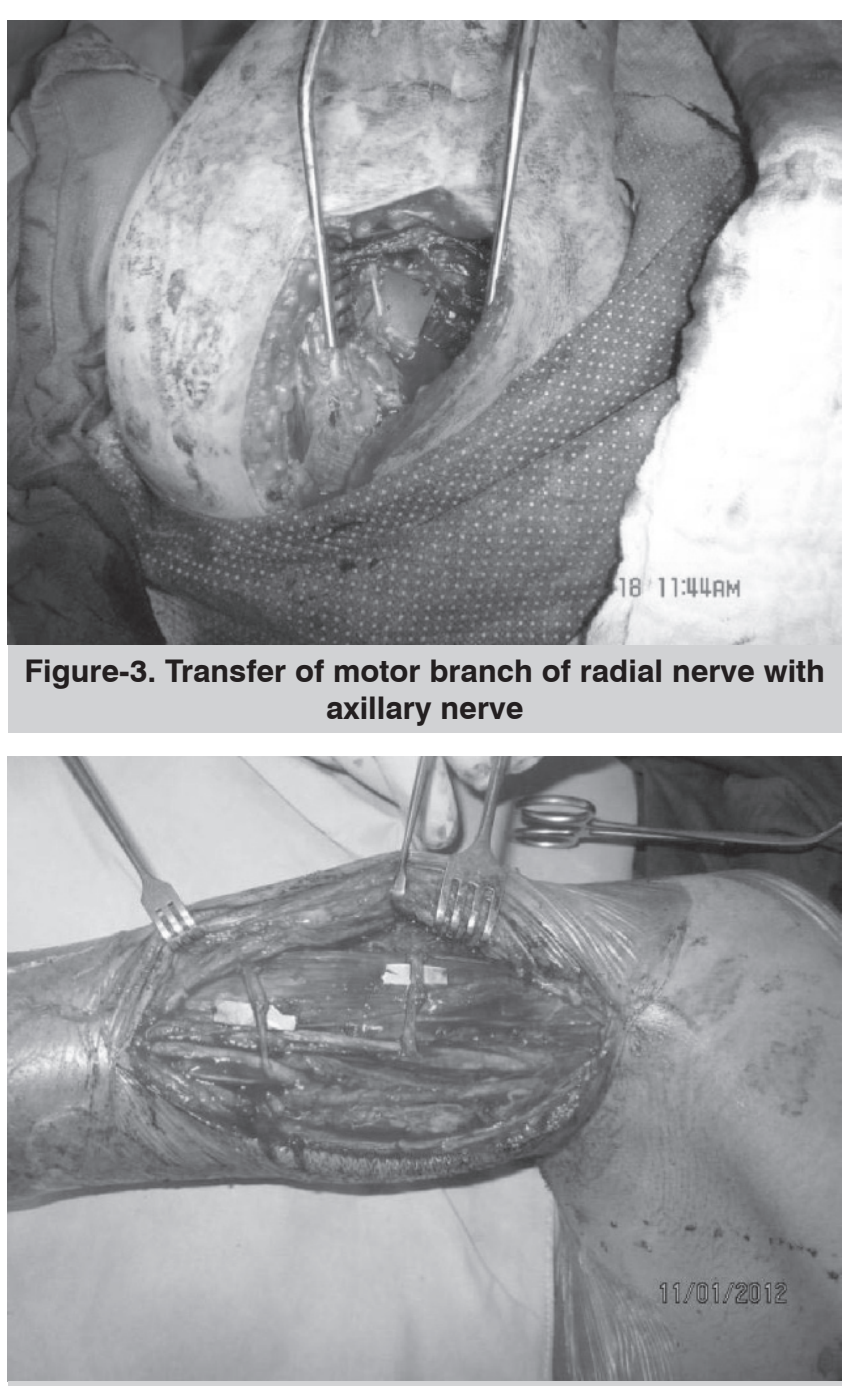

Figure-4. Modified oberlin transfer

\section{DISCUSSION}

In upper brachial plexus injury restoration of elbow flexion and shoulder abduction is the main part of treatment. Joint arthrodesis, tendon and muscle transfer and nerve transfer are different therapeutic options for shoulder stabilization and mobilisation depending upon type and duration of injury. Out of all above mentioned options, nerve transfer has shown superior results in different studies, provided that the patient present within six months after injury.

Nerve transfer for shoulder stabilization varies in two aspects. One aspect is regarding site of nerve transfer. It can be performed either anteriorly by exploring the neck or close to target muscle by a posterior approach. Second aspect is number of nerves reinervated, either suprascapular or axillary alone or both. For first 15 degree shoulder abduction, traditionally, anterior approach was used for the transfer of accessory nerve to suprascapular nerve. The accessory nerve was transacted close to the clavicle in order to spare one or two branches to upper part of trapezius. The drawback of this approach is that it may partially denervate the upper part of trapezius or there are chances of double supra scapular nerve injury resulting in failure of nerve transfer. Recently, there is a trend towards close to target muscle nerve transfer through posterior incision. This approach helps to prevent damage to nerve supply of upper part of trapezius and early recovery of supraspinatus and infraspinatus muscles. Souza et $\mathrm{al}^{11}$ found that better results were obtained when spinal accessory-to-suprascapular nerve transfer was performed using the posterior approach in comparison to anterior neck approach. However, they did not re-innervate the axillary nerve along with suprascapular nerve. The shoulder abduction from 15 to 90 degrees is achieved by axillary nerve supplying the deltoid and teres minor muscles. Again close to target muscle approach has recently been advised for early recovery through the posterior approach. In a study conducted by Cardens -Mejia et $\mathrm{al}^{12}$ in 2008, the results of double nerve transfer for shoulder abduction were much better as compared to single nerve. The drawback of their study was that they mostly used two or three donor nerves to re-innervate only suprascapular nerves. Moreover, in most of the cases they performed nerve transfer through anterior neck incision. We performed double nerve transfer through posterior approach and our results of shoulder abduction were similar to that of Bhandari and colleagues. ${ }^{13}$ Although, they measured the result of shoulder abduction in term of degrees and mean shoulder abduction was 120 degrees which was very much similar to our patients. However, one of the differences was that they performed accessory to suprascapular nerve transfer through anterior approach instead of posterior. ${ }^{13}$

The elbow flexors biceps and brachialis muscles are innervated by the musculocutaneous nerve. 
Traditionally, intercostal or phrenic nerve was transferred to musculocutaneous nerve with variable results. In 1994 Oberlin introduced his technique for restoration of elbow flexion. $10 \%$ of fascicles of ulnar nerve were transferred to motor branch of biceps in the presence of interfasicular connections to prevent any deficit in ulnar distribution after the procedure. ${ }^{14}$ Mackinnon reported double fascicle transfer (modified Oberlin) for elbow flexion. She transferred motor fascicles of both ulnar and median nerve to biceps and brachialis with successful results. ${ }^{10}$ Venkatramani and colleagues found $86 \%$ success rate in case of Oberlin transfer for elbow flexion. ${ }^{15}$ Results were very much similar to our findings. We found $68.8 \%$ M4 strength and $18.8 \%$ M5 strength after double fascicle transfer, one from the ulnar nerve another from the median nerve to brachialis and biceps respectively.

Wilson and colleagues found $97 \%$ elbow flexion after double fascicular transfer to the biceps and brachialis in a series of 29 cases. These outcomes were very much similar to our study in which we found elbow flexion in $97 \%$ of the patients. ${ }^{16}$ Although, most of the studies in traumatic brachial plexus injury showed no residual ulnar and median nerve deficits, but we found partial weakness of median nerve in two patients. In both of them there was loss of index finger flexion and thumb opposition.

However, both the patients recovered within 6 weeks after the surgery and no intervention was done. The possible cause seems to be neuropraxia caused by nerve handling. The advantages are better observed if the nerve transfers are done within 6 months after trauma. The delay in surgery may yield poor results. The results of shoulder abduction were although promising but elbow flexion outcome after modified Oberlin transfer were much more superior as compared to shoulder abduction in our study which were similar to found by Venkatramani and colleagues. ${ }^{15}$

\section{CONCLUSION}

If there is no evidence of recovery up to three months (clinically and by electrophysiological study) early nerve transfer should be considered, ideal time is 3-6 months. Nerve transfer close to target muscle yields superior results. The shoulder stabilizers and abductors should ideally be innervated by double nerve transfer through posterior approach.

Similarly double fasicular transfer (modified Oberlin) should be done for elbow flexion. Although functional improvement in shoulder is not as good as elbow flexion but recovery is phenomenal.

Copyright $@ 13$ Feb, 2020.

\section{REFERENCES}

1. Midha R. Epidemiology of brachial plexus injuries in a multitrauma population. Neurosurgery. 1997 Jun; 40(6): 1182-8.

2. Narakas $A O$. The treatment of brachial plexus injuries. Int Orthop. 1985; 9(1): 29-36.

3. Yeoman PM, Seddon HJ. Brachial plexus injuries: Treatment of the flail arm. J. Bone Joint Surg. (Br.) 1961; 43(3): 493.

4. Brunelli G, Monini L. Neurotization of avulsed roots of the brachial plexus by means of anterior nerves of the cervical plexus. Clin Plast Surg. 1984 Jan; 11(1): 149-52.

5. Allieu Y, Privat JM, Bonnel, F. Paralysis in root avulsion of the brachial plexus: Neurotization by the spinal accessory nerve. Clin. Plast. Surg. 1984 Jan; 11 (1): 133-6.

6. Kotani T, Toshima $\mathrm{Y}$, Mastsuda $\mathrm{H}$, et al. Postoperative results of nerve transposition in brachial plexus injury. Seikei Geka 1971 Oct; 22(11): 963-6.

7. Ray WZ, Murphy KJ, Santosa K, Johnson PJ, Mackinnon SE. Medial pectoral nerve to axillary nerve neurotisation following traumatic brachial plexus injuries: indications and clinical outcomes. Hand (NY) 2012 Mar; 7(1):59-65.

8. Gu YD, Wu MM, Zhen YL, Zhao JA, Zhang GM, Chen DS, Yan JG, Cheng XM. Phrenic nerve transfer for brachial plexus motor neurotization. Microsurgery. 1989; 10(4): 287-9.

9. Tung TH, Mackinnon SE. Nerve Transfer: Indications, techniques and outcomes. J Hand Surg Am. 2010 Feb; 35(2):332-41. 
10. Mackinnon SE, Novak CB, Mackatyn TM, et al. Results of reinnervation of the biceps and brachialis muscles with a double fascicular transfer for elbow flexion. $J$ Hand Surg Am. 2005 Sep; 30(5):978-85.

11. Souza FH, Bernardino SN, Filho HC, Gobbato PL, Martins RS, Martins HA, Silva-Néto RP. Comparison between the anterior and posterior approach for transfer of the spinal accessory nerve to the suprascapular nerve in late traumatic brachial plexus injuries. Acta Neurochir (Wien). 2014 Dec; 156(12):2345-9.

12. Cardenas-Mejia A, O Boyle CP, Chen KT, Chuang DC.I. Evaluation if single-, double- and triple nerve transfer for shoulder abduction in $\mathbf{9 0}$ patients with supraclavicular brachial plexus injury. Plast Reconstr Surg. 2008 Nov; 122(5)1470-8.

13. Bhandari PS, Sadhotra LP, Bhargava P, AS Bath AS, Mukherjee MK, Bhatti TS, Maurya S. Multiple nerve transfers for the reanimation of shoulder and elbow functions in irreparable C5, C6 and upper truncal lesions of the brachial plexus. IJNT 2008 Dec; 5(2):95104.
14. Oberlin C, Beal D, Leechavengvongs S, Salon A, Dauge MC, Sarry JJ. Nerve transfer to biceps muscle using part of ulnar nerve for C5-C6 avulsion of the brachial plexus: Anatomical study and report of four cases. $J$ Hand Surg Am 1994; 3(2):232-237.

15. Venkatramani $H$, Bhardwaj $P$, Faruquee SR, Sabapathy $S R$. Functional outcome of nerve transfer for restoration of shoulder and elbow function in upper brachial plexus injury. J Brachial Plex Peripher Nerve Inj. 2008 May; 3: 15.

16. Ray ZW, Pet MA, Yee A, Mackinnon SE. Double fascicular nerve transfer to the biceps and brachialis muscles after brachial plexus injury: Clinical outcomes in a series of 29 cases. J Neurosurg 2011 Jun; 114(6):1520-28.

\begin{tabular}{|c|c|c|c|}
\hline \multicolumn{4}{|c|}{ AUTHORSHIP AND CONTRIBUTION DECLARATION } \\
\hline Sr. \# & Author(s) Full Name & Contribution to the paper & Author(s) Signature \\
\hline 1 & Husnain Khan & $\begin{array}{l}\text { Conceived \& designed the } \\
\text { analysis. }\end{array}$ & 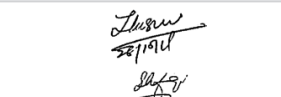 \\
\hline 2 & Muhammad Shafique & Collected the data. & ज्ञाये \\
\hline 3 & Zahid Iqbal Bhatti & Wrote the paper & $\frac{2 \pi}{2 \pi / 02}$ \\
\hline 4 & Tehseen Ahmad Cheema & Performed the analysis. & 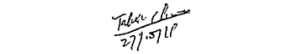 \\
\hline
\end{tabular}

